\title{
Presepsin Levels in Experimental Sepsis and Massive Bowel Resection Models in Rats
}

\author{
OZLEM UNAY-DEMIREL ${ }^{1}$, SEYDA IGNAK $^{2}$, TANER ORUG $^{3}$ and MERAL YUKSEL ${ }^{4}$ \\ ${ }^{1}$ Department of Biochemistry, Medical Park Goztepe Hospital, \\ Bahcesehir University School of Medicine, Istanbul, Turkey; \\ ${ }^{2}$ Department of Medical Biology, Bahcesehir University School of Medicine, Istanbul, Turkey; \\ ${ }^{3}$ Department of Surgery, Medicana Hospital, Istanbul, Turkey; \\ ${ }^{4}$ Department of Medical Laboratory Techniques, \\ Vocational School of Health Services, Marmara University, Istanbul, Turkey
}

\begin{abstract}
Background/Aim: Presepsin is a useful biomarker for diagnosing sepsis. This study aimed to investigate the relationship between oxidative stress and presepsin levels in animal models. Materials and Methods: Sprague-Dawley rats were used for cecal ligation and puncture $(C L P)$ and to generate massive bowel resection (MBR) models. Trunk blood was collected for analysis of presepsin. Liver and intestinal tissue samples were taken to determine oxidative stress parameters. Results: Presepsin levels in MBR and $C L P$ sepsis models were higher than those in control groups. Reactive oxygen and nitrogen species (RONS) and malondialdehyde levels were increased in the liver and small intestine of rats in both models, whereas glutathione levels were decreased. Conclusion: Presepsin levels and RONS may be released by the same mechanism which is closely associated with the progression of sepsis and inflammation in both CLP and MBR models.
\end{abstract}

Sepsis is a systemic inflammatory response to infections that, despite modern treatments, remains a life-threatening challenge in medicine. Although bacterial culture is a gold standard test for the diagnosis of sepsis, it is time-consuming and false-negative results have been reported because of previous and concomitant antibiotic therapy. Early diagnosis

This article is freely accessible online.

Correspondence to: Ozlem Unay Demirel, MD, Asst. Prof., Bahcesehir University, School of Medicine, Goztepe Medical Park Hospital Laboratory and Transfusion Center, 23, Nisan Sokak E-5 Uzeri 34732 Kadıkoy, Istanbul, Turkey. Tel: +90 2164684292, Fax: +90 2164684084, e-mail: ozlemunay@yahoo.com

Key Words: Sepsis, presepsin, oxidative stress, massive bowel resection syndrome, cecal ligation and perforation. is important to prevent delay in accurate treatment and decrease mortality incidence, which can reach up to $5 \%$ to $10 \%(1-3)$. It has been shown that free radicals [reactive oxygen species (ROS) and reactive nitrogen species (RNS)] affect the onset, progression, and outcome of sepsis. ROS and RNS are antimicrobial agents produced by neutrophils and monocyte/macrophages to destruct microorganisms during phagocytosis. Also, NF-kappaB, which tightly regulates the expression of proinflammatory cytokines, is modulated by ROS. It is well known that excessive production of proinflammatory cytokines, ROS and RNS, has detrimental cytotoxic effects, which lead to multiorgan system failure and mortality (4-6). Superoxide anion, nitric oxide, hydroxyl radical, and hydrogen peroxide are important members of ROS/RNS (4). There is an antioxidant system, which acts by buffering ROS/RNS production so that healthy individuals keep the balance between oxidants and antioxidants level. Whenever there is an impairment in oxidant and antioxidant level, signal transduction pathways change leading to disturbed cellular homeostasis, and possibly to organ dysfunction and multiorgan failure $(1,6,7)$. It has been reported that patients who died from severe sepsis showed deficiency of antioxidants, whereas sepsis survivors had increased levels of antioxidants $(8,9)$. There are many reported biomarkers for sepsis, such as C-reactive protein, procalcitonin, and interleukins, which aid in early diagnosis. In 2004, Yaegashi et al. found a new biomarker which is the soluble subtype of sCD14 (sCD14-ST) named presepsin (10). The innate immunity is the first barrier against bacteria, and partially relies on the membrane receptor CD14 (11). CD14 is found on different cells including in neutrophils, monocytes, macrophages, and $\mathrm{B}$ cells, that possess high-affinity receptors for lipopolysaccharide-lipopolysaccharide binding protein (LPS-LBP). CD14 induces signal transduction through this receptor via CD14-mediated activation of toll-like receptors, which leads to inflammatory gene expression and activation 
Table I. Experimental design of groups.

\begin{tabular}{lll}
\hline Groups & $\mathrm{n}$ & Protocol \\
\hline Sham MBR & 7 & $\begin{array}{l}\text { Sham-operated for massive bowel resection (the bowel was cut at } 10 \mathrm{~cm} \text { proximal to ileocecal } \\
\text { valve and re-anastomosed without resection) }\end{array}$ \\
MBR & 7 & $\begin{array}{l}\text { Massive small bowel resection (80\% resection of measured small bowel length) } \\
\text { Sham CLP }\end{array}$ \\
CLP & 7 & Sham-operated for cecal ligation and puncture (laparatomy and cecal exposure without any more manipulation) \\
\end{tabular}

of inflammatory pathways. The glycoprotein CD14 has two forms: membrane-bound (mCD14) or circulating soluble form (sCD14). Presepsin can originate either from the detachment of membrane-bound CD14 (mCD14) expressed by phagocytes or cell secretion $(4,12,13)$. It has been proposed that presepsin is a possible acute-phase protein produced by hepatocytes, besides the protease-mediated shedding from leucocytes. Presepsin is cleaved by plasma proteases $(12,13)$. Presepsin has been suggested as a novel sepsis biomarker which can be detected easier than mCD14 in the blood and is a suitable prognostic factor in the diagnosis of sepsis (11-13).

The link between free radical generation and presepsin in sepsis is not known yet. We hypothesized that active neutrophils/monocytes/macrophages secrete RONS while they produce presepsin. We aimed to investigate the possible relation between oxidative stress (malondialdehyde, reactive oxygen, and nitrogen species), antioxidant molecules (glutathione), and presepsin levels in the well-known experimental sepsis rat model generated by cecal ligation and perforation (CLP) and in another possible sepsis model which is the massive bowel resection (MBR) model.

\section{Materials and Methods}

Experimental groups. All experiments were conducted in accordance with the national guidelines for laboratory animal care and use, and the study protocol was approved by the Ethical Committee of Marmara University. Female Sprague-Dawley rats (weighing 250-300 g), were kept in a room at a temperature of $22 \pm 2^{\circ} \mathrm{C}$, with $12 \mathrm{~h} / 12 \mathrm{~h}$ light and dark cycle, and fed with standard pellet chow and water ad libitum.

Rats were assigned randomly into four groups. Group 1 received a massive small bowel resection (MBR); group 2 received sham MBR and served as the control group without a resection. Group 3 received a cecal ligation and perforation (CLP) after laparotomy, and group 4 received sham CLP and served as the control without a cecal ligation after laparotomy (Table I).

Surgical procedures. After overnight fasting, surgical procedures were performed. Animals were anesthesized with intramuscular administration of ketamine hydrochloride at $50 \mathrm{mg} / \mathrm{kg}$ (Ketalar 50 $\mathrm{mg} / \mathrm{ml}$, Pfizer, Luleburgaz, Turkey) and Xylazine-hydrochloride at $10 \mathrm{mg} / \mathrm{kg}$ (Alfazyne, Alfasan Int. BV Woerden, The Netherlands). The abdomen of rats in the massive bowel resection group (group
1) was cut with a midline incision (3-4 cm) under sterile conditions, and approximately $80 \%$ of the small intestine was transected leaving about 5 to $6 \mathrm{~cm}$ from the ligament of Treitz and the ileocecal valve. The bowel ends were sutured together with 60 Prolene. Sterile $10 \mathrm{ml}$ saline which was kept warm was administered into the peritoneal cavity for postoperative fluid balance, and 4-0 silk was used to suture the abdomen. Rats in the sham MBR received a laparotomy operation with enterotomy and anastomosis was performed (14).

Rats in the cecal ligation and perforation (CLP) group (group 2) were treated as described by Fujimura $\mathrm{N}$ and Dejager L et al. (15, 16). The rats received a small midline incision under ether anesthesia, and cecum was joined just proximal to the ileocecal valve with 3-0 silk to maintain intactness of intestines. The antimesenteric cecal surface was punctured by an 18-gauge needle at two sites which were $1 \mathrm{~cm}$ distant to each other, and the cecum was kindly squeezed until the feces were forced out. The laparotomy was closed with 3-0 silk. All rats received injections with saline ( $3 \mathrm{ml} / 100 \mathrm{~g}$ body weight) through the subcutaneous route to resuscitate postoperatively. The rats in the sham operated for CLP group underwent only laparotomy and cecal exposure without any other manipulation.

Forty-eight $\mathrm{h}$ after operation, the rats underwent decapitation. After decapitation, trunk blood was collected, centrifuged, and serum was kept at $-20^{\circ} \mathrm{C}$ for analysis of presepsin levels. Liver and intestinal tissue samples were immediately collected for biochemical evaluation of glutathione (GSH), malondialdehyde (MDA) and RONS.

Determination of malondialdehyde and glutathione. Tissue samples were homogenized with ice-cold $0.067 \%$ (w/v) TCA for analysis of MDA and GSH levels. After centrifugation, the supernatant was mixed with thiobarbituric acid and boiled at $95^{\circ} \mathrm{C}$ for $30 \mathrm{~min}$. For the analysis of MDA, the levels of thiobarbituric acid reactive substances (TBARS) which are the byproducts of lipid peroxidation were monitored as described by Beuge et al. (17). The extinction coefficient of $1.56 \times 10^{5}$ $\mathrm{M}^{-1} \mathrm{~cm}^{-1}$ was used to determine MDA equivalents and results were expressed as nmol MDA/g tissue. GSH was determined by using the Ellman's procedure with modifications. In summary, supernatants of small intestinal and liver samples were added to $\mathrm{Na}_{2} \mathrm{HPO}_{4} \cdot 2 \mathrm{H}_{2} \mathrm{O}$ solution and dithio-bis nitrobenzoic acid was added. Absorbance was monitored instantly after mixing at $412 \mathrm{~nm}$. GSH levels were determined by the use of the extinction coefficient of $13,600 \mathrm{M}^{-1}$ $\mathrm{cm}^{-1}$. Results were expressed as $\mu \mathrm{mol} \mathrm{GSH} / \mathrm{g}$ tissue (18).

Determination of RONS. The chemiluminescence (CL) method was used to assess RONS levels in tissue samples. Liver or small intestine tissue pieces (approximately $25-30 \mathrm{mg}$ ) were put into 
Table II. Presepsin levels in the groups analysed.

\begin{tabular}{llcc}
\hline Group & $\begin{array}{c}\text { Mean } \\
(\mathrm{pg} / \mathrm{ml})\end{array}$ & $\begin{array}{c}\text { Standard error } \\
\text { of mean }\end{array}$ & $p$-Value \\
\hline Sham CLP & 240 & 80.106 & $p<0.01$ \\
CLP & 528 & 121.7 & \\
Sham MBR & 286.3 & 96.03 & $p<0.05$ \\
MBR & 850.6 & 328.1 & \\
\hline
\end{tabular}

CLP: Cecal ligation and perforation; MBR: massive small bowel resection.

three test tubes containing phosphate buffered saline and HEPES (pH: 7.8). Counts of CL were analyzed afterwards by the addition of $0.2 \mathrm{mmol} / 1$ lucigenin that is selective for superoxide radical; 0.2 $\mathrm{mmol} / \mathrm{l}$ luminol which measures hydroxyl radical, hydrogen peroxide and hypochloride and $0.4 \mathrm{mmol}$ potassium carbonate, 9 mmol desferrioxamine, $0.2 \mathrm{mmol}$ hydrogen peroxide and $50 \mathrm{mmol}$ luminol sodium salt for the determination of nitric oxide release. Analyses were performed via a luminometer (Mini Lumat LB 9509, EG\&G Berthold, Bad Wildbad, Germany) and results were recorded at $1 \mathrm{~min}$ intervals for $5 \mathrm{~min}$ and normalized to $\mathrm{mg}$ tissue weight. Data was given as area under the curve of relative light units (rlu) (19).

Determination of presepsin levels. Rat presepsin levels were determined by Aviva Systems Biology CD14 ELISA Kit (San Diego, CA, USA), which is based on a standard sandwich enzyme linked immuno-sorbent assay (ELISA). The results were expressed as $\mathrm{pg} / \mathrm{ml}$.

Statistics. Instat program was used for statistical analysis. Descriptive statistics of categorical variables are given with frequency and percent where continuous variables are represented by the mean and standard error of mean values. One-way analysis of variance (ANOVA) test was used for test of normality. TukeyKramer multiple comparison test was used to test significant difference for dependent variables at different times. Spearman's correlations analyses between presepsin and RONS, MDA and GSH were used. For all statistical analyses, a $p$-value below 0.05 was considered to indicate statistically significant differences.

\section{Results}

Presepsin levels in the MBR $(850.6 \pm 328.1 \mathrm{pg} / \mathrm{ml})$ and CLP sepsis models at 48 th $\mathrm{h}(528 \pm 121.7 \mathrm{pg} / \mathrm{ml})$ were significantly higher than those in the sham-operated control rats $(286.3 \pm 96.03 \mathrm{pg} / \mathrm{ml} ; p<0.05$ vs. $240 \pm 80.106 \mathrm{pg} / \mathrm{ml} ; p<0.01$, respectively) (Table II).

We also observed that ROS production, nitric oxide (NO) release and MDA levels were increased significantly in the liver and small intestine of rats in both CLP and MBR models whereas GSH levels were decreased, indicating oxidative stress $(p<0.05)$. Comparisons of MDA and GSH levels in the small intestine and liver among groups are shown in Figure 1. The levels of luminol and lucigenin levels that indicate the levels of superoxide radical and

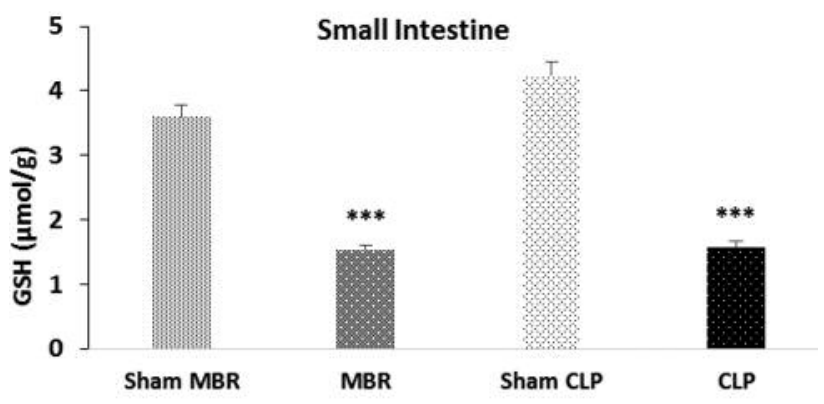

Liver

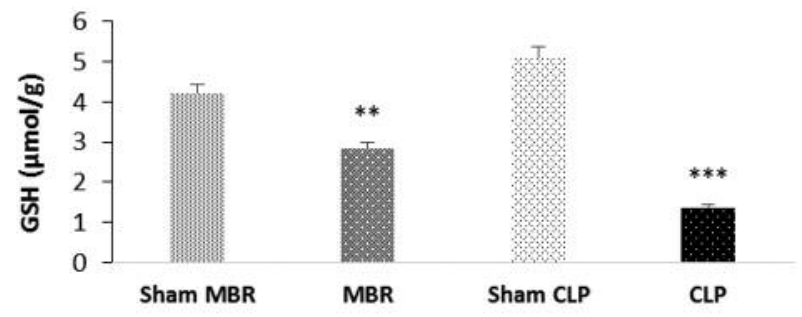

Small Intestine

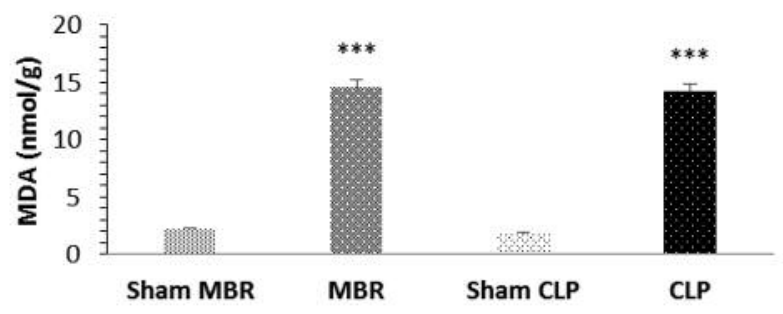

Liver

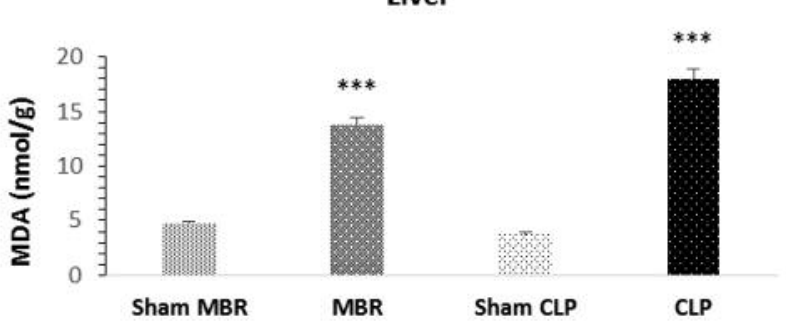

Figure 1. Glutathione and malondialdehyde levels in the liver and small intestine of MBR and CLP rat models. Both groups were compared with their sham-operated control groups; $* p>0.05, * * p<0.05$, ${ }^{* * *} p<0.001$. CLP: Cecal ligation and perforation; MBR: massive small bowel resection.

hydroxyl radical, hydrogen peroxide and hypochloride as well as the levels of nitric oxide release are shown in Figure 2. We did not find a correlation between presepsin and oxidative stress parameters such as RONS, MDA and GSH levels in both experimental rat models $(p>0.05)$. 

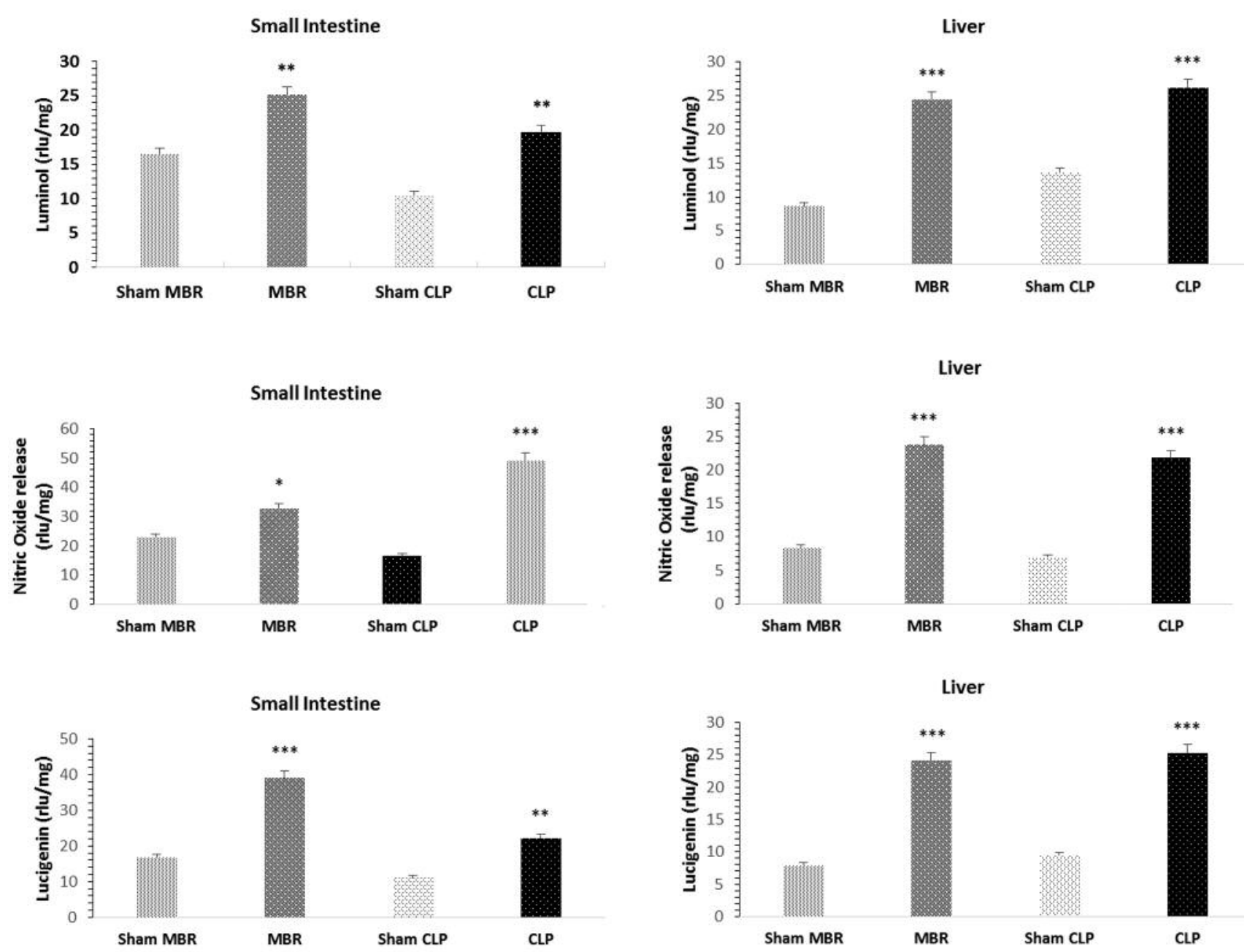

Figure 2. Luminol, lucigenin and nitric oxide levels in the liver and small intestine of MBR and CLP rat models. Both groups were compared with their sham-operated control groups; $* p>0.05, * * p<0.05, * * * p<0.001$. CLP: Cecal ligation and perforation; MBR: massive small bowel resection.

\section{Discussion}

We examined the possible relation between oxidative stress, glutathione and presepsin levels in a proinflammatory MBR model and a well-known sepsis model CLP to shed light on the pathophysiology of sepsis which may be helpful to develop an early diagnostic marker such as presepsin. It is obvious that neutrophils play a major role in cellular signaling events related with inflammatory and immune responses. Neutrophils produce large amounts of ROS and RNS by the oxidant generating systems like NADPH oxidase and nitric oxide synthase. These oxidants are highly potent and have the ability to kill pathogens directly. Oxidants can modulate gene expression by effecting transcription factors such as NF-kB that is closely associated with expression of genes which play a role in the acute inflammatory response. It is known that several cytokines, adhesion molecules and acute-phase proteins are involved in this acute response. Moreover NF-kB has a role in the transcriptional regulation of some cytokines, such as IL-1, IL-6, IL-8, which are produced by neutrophils during the inflammatory process. It is known that cytokines like TNF and IL-1 can lead directly to NF-kB activation and TNF-mediated NF-kB activation is influenced by production of ROS $(6,20-22)$. Oxidative stress which regulates the activation of NF-kB is observed in sepsis patients and therefore, NF-kB-mediated increases in the expression of cytokines is also detected in these patients (23).

Although the mechanism of secretion of presepsin is currently unclear, it is has been suggested that it depends on phagocytosis $(11,13)$. Moreover, RONS are antimicrobial 
agents produced by neutrophils and monocyte/macrophages to destruct microorganisms during phagocytosis $(5,6)$. Therefore, both presepsin and RONS production may be triggered by the same mechanism in sepsis.

As far as we know, there is no publication on this subject. The presepsin levels in MBR and CLP rat models were significantly higher than their sham-operated controls. We also observed that RONS release was increased significantly in the liver and small intestine of rats in both CLP and MBR model, whereas GSH levels were decreased indicating oxidative stress. Consistent with the literature, MDA levels, an index of lipid peroxidation, were increased significantly substantiating further the presence of oxidative stress.

In our study, we used two animal models: An experimental MBR model and the commonly used CLP model for sepsis. CLP is the most frequently used and is considered as a gold standard model for sepsis research (16). Short bowel syndrome is defined as malabsorption after resection of small intestine. The most common complications which cause death during the MBR are sepsis and liver failure (24). There are several publications which indicate the role of parenteral nutrition (PN) in MBR associated inflammation $(25,26)$. Clinical or experimental studies that have investigated the mechanism of sepsis in MBR patients without PN suggested that MBR without PN is a proinflammatory state and the increased production of proinflammatory cytokines may result in liver damage in sepsis $(27,28)$. It has been shown that massive bowel resection alone can cause liver pathologies but the underlying molecular mechanism has not been elucidated (14). However, O'Brien et al. have proposed that massive small-bowel resection may cause bacterial translocation in animals which may lead to repeating episodes of sepsis but the precise mechanism for bacterial translocation after massive small bowel resection is currently unknown (29). In our study, we obtained similar findings in terms of presepsin, oxidant and antioxidant parameters in both MBR group and the CLP sepsis group. There is a small number of studies on presepsin in experimental animal models. A study of sepsis in rabbit models has shown that presepsin levels are increased in the CLP sepsis model, but no increase was observed in LPS-induced sepsis model. In that study it was suggested that SCD14-ST production is dependent on phagocytosis and cathepsin D is one of the enzymes responsible for fragmentation of CD14 (30).

In another rabbit CLP sepsis model, Nakamura et al. have shown that plasma sCD14 levels increased earlier than biomarkers, such as IL-6, D-dimer, and bacteria in the blood, and suggested that SCD14-ST is an early diagnostic tool for initiating anti-sepsis therapy (31). In our study, we observed increased presepsin levels both in CLP and MBR models, which were significantly higher than those in sham-operated control rats. It is known that CLP-induced sepsis is associated with a remarkable inflammatory response and the production of ROS (32). We also observed significantly higher ROS and RNS levels in the liver and intestinal tissues of CLP and MBR experimental groups than those in shamoperated control tissues. However, there was no correlation between presepsin and RONS levels.

The pathogenesis of sepsis, which is a serious and lifethreatening systemic illness, depends on the interaction between various microbial and host factors. Early diagnosis of sepsis has utmost importance due to the huge economic burden needed for long-term hospital stay and use of broad-spectrum antibiotics. Moreover, there can be resistance to antibiotics. It has also been shown that early diagnosis of sepsis can be lifesaving with the use of appropriate treatment strategy. Several biomarkers have been developed for the diagnosis of sepsis such as procalcitonin and C-reactive protein, but they have limitations in their use like sensitivity and specificity problems (2). Furthermore, sCD14-subtypes (presepsin) were discovered as a new biomarker for sepsis in 2004 and several studies have been conducted to delineate the importance of presepsin for early diagnosis and follow up of sepsis $(13,33,34)$. It is well known that presepsin is produced in response to phagocytosis of bacteria in infections, however, it is not yet clear which humoral and cellular factors are responsible for the production of presepsin $(13,35)$.

Several limitations of this study should be acknowledged. Although we found that both presepsin and RONS levels increase during sepsis, we could not show significant correlation among presepsin and RONS. Also, evaluating the concentration of specific proinflammatory mediators, such as IL-6, IL-1 beta, and TNF-alpha in addition to presepsin may be helpful to elucidate whether presepsin and RONS use the same or related pathways leading to sepsis.

This is the first study, to our knowledge, examining presepsin levels in experimental rat models. Our results suggested that presepsin levels and RONS may be released by the same mechanism, which is closely associated with the progression of sepsis and inflammation in both experimental CLP and MBR rat models. Future studies should be performed to determine the levels of several cytokines in experimental animal models and delineate their role in sepsis.

\section{Conflicts of Interest}

The Authors declare that there are no conflicts of interest regarding this study.

\section{Authors' Contributions}

O.UD, T.O, S.I and M.Y participated in the design of study, O.UD, M.Y carried out biochemical analysis, T.O performed experimental precedures on animals, M.Y, O.UD, S.I performed the statistical analysis, S.I and O.UD evaluated the results and wrote the paper. All Authors read and approved the final article. 


\section{Acknowledgements}

The Authors received no financial support for this research.

\section{References}

1 Andrades ME, Ritter C and Dal-Pizzol F: The role of free radicals in sepsis development. Front Biosci 1: 277-287, 2009. PMID: 19482645.

2 Bauer M and Reinhart K: Molecular diagnostics of sepsis--where are we today? Int J Med Microbiol 300: 411-413, 2010. PMID: 20510650. DOI: 10.1016/j.ijmm.2010.04.006

3 Sankar V and Webster NR: Clinical application of sepsis biomarkers. J Anesth 27: 269-283, 2013. PMID: 23108494. DOI: $10.1007 / \mathrm{s} 00540-012-1502-7$

4 Kaymak C, Kadioglu E, Ozcagli E, Osmanoglu G, Izdes S, Agalar C, Basar H and Sardas S: Oxidative DNA damage and total antioxidant status in rats during experimental gram-negative sepsis. Hum Exp Toxicol 27: 485-491, 2008. PMID: 18784201. DOI: $10.1177 / 0960327108088972$

5 Kumar S, Gupta E, Kaushik S, Srivastava VK, Mehta SK and Jyoti A: Evaluation of oxidative stress and antioxidant status: correlation with the severity of sepsis. Scand J Immunol 87: e12653, 2018. PMID: 29484685. DOI: 10.1111/sji.12653

6 Fialkow L, Wang Y and Downey GP: Reactive oxygen and nitrogen species as signaling molecules regulating neutrophil function. Free Radic Biol Med 42: 153-164, 2007. PMID: 17189821. DOI: $10.1016 /$ j.freeradbiomed.2006.09.030

7 Georgieva E, Ivanova D, Zhelev Z, Bakalova R, Gulubova M and Aoki I. Mitochondrial dysfunction and redox imbalance as a diagnostic marker of "free radical diseases". Anticancer Res 37: 5373-5381, 2017. PMID: 28982845. DOI: 10.21873/anticanres. 11963

8 Karapetsa M, Pitsika M, Goutzourelas N, Stagos D, Tousia Becker A and Zakynthinos E: Oxidative status in ICU patients with septic shock. Food Chem Toxicol 61: 106-111, 2013. PMID: 23542126. DOI: 10.1016/j.fct.2013.03.026

9 Cowley HC, Bacon PJ, Goode HF, Webster NR, Jones JG and Menon DK: Plasma antioxidant potential in severe sepsis: a comparison of survivors and nonsurvivors. Crit Care Med 24: 1179-1183, 1996. PMID: 8674332. DOI: 10.1097/00003246199607000-00019

10 Yaegashi Y, Shirakawa K, Sato N, Suzuki Y, Kojika M, Imai S, Takahashi G, Miyata M, Furusako S and Endo S: Evaluation of a newly identified soluble CD14 subtype as a marker for sepsis. J Infect Chemother 11: 234-238, 2005. PMID: 16258819. DOI: 10.1007/s10156-005-0400-4

11 Chenevier-Gobeaux C, Borderie D, Weiss N, Mallet-Coste T and Claessens YE: Presepsin (sCD14-ST), an innate immune response marker in sepsis. Clin Chim Acta 450: 97-103, 2015. PMID: 26164388. DOI: 10.1016/j.cca.2015.06.026

$12 \mathrm{Wu}$ CC, Lan HM, Han ST, Chaou CH, Yeh CF, Liu SH, Li CH, Blaney GN, Liu ZY and Chen KF: Comparison of diagnostic accuracy in sepsis between presepsin, procalcitonin, and C-reactive protein: a systematic review and meta-analysis. Ann Intensive Care 7: 91, 2017. DOI: 10.1186/s13613-017-0316-Z

13 Zou Q, Wen W and Zhang XC: Presepsin as a novel sepsis biomarker. World J Emerg Med 5: 16-19, 2014. PMID: 25215141. DOI: 10.5847/wjem.j.1920-8642.2014.01.002
14 Bostanoğlu A, Oruğ T, Yıldız BD, Işık S, Zengin Nİ, Evren E and Saydam GS: Experimental study on the effects of massive bowel resection on liver function and hepatocyte apoptosis. Turk J Gastroenterol 25: 674-677, 2014. PMID: 25599780. DOI: $10.5152 /$ tjg. 2014.5229

15 Fujimura N, Sumita S, Narimatsu E, Nakayama Y and Shitinohe: Effects of isoproterenol on diapragmatic contractility in septic peritonitis. Am J Respir Crit Care Med 161: 440-446, 2000. PMID: 10673183. DOI: 10.1164/ajrccm.161.2.9904044

16 Dejager L, Pinheiro I, Dejonckheere E and Libert C: Cecal ligation and puncture: the gold standard model for polymicrobial sepsis? Trends Microbiol 19: 198-208, 2011. PMID: 21296575. DOI: $10.1016 / \mathrm{j} . \mathrm{tim} .2011 .01 .001$

17 Beuge JA and Aust SD: Microsomal lipid peroxidation. Methods Enzymol 52: 302-311, 1978. PMID: 672633. DOI: 10.1016/ s0076-6879(78)52032-6

18 Beutler E: Reduced Glutathione (GSH). In: Red Blood Cell Metabolism: A Manual of Biochemical Method. Bergmeyen HV (ed.). New York, Grune and Stratton, pp. 112-114, 1975.

19 Haklar U, Yuksel M, Velioglu A, Turkmen M, Haklar G and Yalcin AS: Oxygen radicals and nitric oxide levels in chondral or meniscal lesions or both. Clin Orthop Relat Res 403: 135-142, 2002. PMID: 1236001919.

20 Touyz RM: Reactive oxygen species as mediators of calcium signaling by angiotensin II: implications in vascular physiology and pathophysiology. Antioxid Redox Signal 7: 1302-1314, 2005. PMID: 16115036. DOI: 10.1089/ars.2005.7.1302

21 Korhonen R, Lahti A, Kankaanranta H and Moilanen E: Nitric oxide production and signaling in inflammation. Curr Drug Targets Inflamm Allergy 4: 471-479, 2005. PMID: 16101524.

22 Heneberg P and Dráber P: Regulation of cys-based protein tyrosine phosphatases via reactive oxygen and nitrogen species in mast cells and basophils. Curr Med Chem 12: 1859-1871, 2005. PMID: 16101506. DOI: 10.2174/0929867054546636

23 Macdonald J, Galley HF and Webster NR: Oxidative stress and gene expression in sepsis. Br J Anaesth 90: 221-232, 2003. PMID: 12538380 . DOI: 10.1093/bja/aeg034

24 Schalamon J, Mayr JM and Höllwarth ME: Mortality and economics in short bowel syndrome. Best Prac Rs Clin Gastroenterol 17: 931-942, 2003. PMID: 14642858.

25 Kurkchubasche AG, Smith SD and Rowe MI: Catheter sepsis in short-bowel syndrome. Arch Surg 127: 21-24, 1992. PMID: 1734846. DOI: 10.1001/archsurg.1992.01420010027004

26 Alverdy JC, Aoys E and Moss GS: Total parenteral nutrition promotes bacterial translocation from the gut. Surgery 104: 185190, 1988. PMID: 313562526.

27 Asensio AB, García-Urkia N, Aldazabal P, Bachiller P, GarcíaArenzana JM and Eizaguirre I: Incidence of bacterial translocation in four different models of experimental short bowel syndrome. Cir Pediatr 16: 20-25, 2003. PMID: 12793289.

28 Aprahamian CJ, Chen M, Yang Y, Lorenz RG and Harmon CM: Two-hit rat model of short bowel syndrome and sepsis: independent of total parenteral nutrition, short bowel syndrome is proinflammatory and injurious to the liver. J Pediatr Surg 42: 992997, 2007. PMID: 17560208. DOI: 10.1016/j.jpedsurg.2007.01.071

29 O'Brien DP, Nelson LA, Kemp CJ, Williams JL, Wang Q, Erwin $\mathrm{CR}$, Hasselgren PO and Warner BW: Intestinal permeability and bacterial translocation are uncoupled after small bowel resection. J Pediatr Surg 37: 390-394, 2002. PMID: 11877654. DOI: $10.1053 /$ jpsu.2002.30807 
30 Naitoh K, Shirakawa K, Hirose J, Nakamura M, Takeuchi T, Hosaka Y and Furusako S: The new sepsis marker, sCD14-ST (Presepsin): induction mechanism in the rabbit sepsis models. Crit Care 14: Poster-19, 2010.

31 Nakamura M, Takeuchi T, Naito K, Shirakawa K, Hosaka Y, Yamasaki F and Furusako S: Early elevation of plasma soluble CD14 subtype, a novel biomarker for sepsis, in a rabbit cecal ligation and puncture model. Crit Care 12: Poster-194, 2008.

32 Li S, Wu H, Han D, Ma S, Fan W, Wang Y, Zhang R, Fan M, Huang Y, Fu X and Cao F: A novel mechanism of mesenchymal stromal cell-mediated protection against sepsis: restricting inflammasome activation in macrophages by increasing mitophagy and decreasing mitochondrial ROS. Oxid Med Cell Longev 2018: 3537609, 2018. PMID: 29636842. DOI: 10.1155/ 2018/3537609

33 Bellos I, Fitrou G, Pergialiotis V, Thomakos N, Perrea DN and Daskalakis G: The diagnostic accuracy of presepsin in neonatal sepsis: a meta-analysis. Eur J Pediatr 177: 625-632, 2018. PMID: 29476345. DOI: 10.1007/s00431-018-3114-1
34 Yang HS, Hur M, Yi A, Kim H, Lee S and Kim SN: Prognostic value of presepsin in adult patients with sepsis: systematic review and meta-analysis. PLoS One 13: e0191486, 2018. PMID: 29364941. DOI: 10.1371/journal.pone.0191486

35 Bas S, Gauthier BR, Spenato U, Stingelin S and Gabay C: CD14 is an acute-phase protein. J Immunol 172: 4470-4479, 2004. PMID: 15034063. DOI: 10.4049/jimmunol.172.7.4470
Received October 10, 2019

Revised October 24, 2019

Accepted October 25, 2019 